\title{
Poverty index with time-varying consumption and income distributions
}

\author{
Amit K. Chattopadhyay* \\ Aston University, Mathematics, Birmingham B4 7ET, United Kingdom \\ T. Krishna Kumar ${ }^{\dagger}$ \\ Rockville-Analytics, Rockville, Maryland 20850, USA \\ Sushanta K. Mallick \\ (Received 29 June 2016; revised manuscript received 22 November 2016; published 6 March 2017)
}

School of Business and Management, Queen Mary University of London, Mile End Road, London E1 4NS, United Kingdom

\begin{abstract}
Starting from a stochastic agent-based model to represent market exchange in a developing economy, we study time variations of the probability density function of income with simultaneous variation of the consumption deprivation (CD), where $\mathrm{CD}$ represents the shortfall in consumption from the saturation level of an essential commodity, cereal. Together, these two models combine income-expenditure-based market dynamics with time variations in consumption due to income. In this new unified theoretical structure, exchange of trade in assets is only allowed when the income exceeds consumption-deprivation while CD itself is endogenously obtained from a separate kinetic model. Our results reveal that the nature of time variation of the CD function leads to a downward trend in the threshold level of consumption of basic necessities, suggesting a possible dietary transition in terms of lower saturation level of food-grain consumption, possibly through an improvement in the level of living. The new poverty index, defined as $\mathrm{CD}$, is amenable to approximate probabilistic prediction within a short time horizon. A major achievement of this work is the intrinsic independence of the poverty index from an exogenous poverty line, making it more objective for policy formulation as opposed to existing poverty indices in the literature.
\end{abstract}

DOI: 10.1103/PhysRevE.95.032109

\section{INTRODUCTION}

There is poverty among the traditional studies on economics of poverty, as most of them are deprived of any direct link to the commonly understood notion of poverty as consumption deprivation of essential goods and services. In this study, we eliminate that deficit by linking the poverty index directly to microeconomic behavior of individuals at low levels of consumption of essential commodities within a market exchange mechanism. Our study is in the tradition of Engel $[1,2]$, who was a pioneer in undertaking the living conditions of the poor by observing the pattern of their consumption and expenditure. Although Deaton also established a link between poverty and consumption, for which he was awarded the Nobel Prize in economics [3], our study differs in a significant way. While Deaton and most others [4] use an exogenous poverty line to measure poverty, we deviate from that tradition by defining and measuring poverty without a poverty line.

Market exchange involves exchange of commodities between economic agents who agree to a bartering network between themselves. Within this network, there are pockets of individuals or families whose rate of flow of income derived from exchanging their own human skills in the market place is not adequate to meet their necessities. Such people are referred to as poor and their state of living or consumption is called poverty. To understand the mechanics of income and consumption of such individuals, and the associated measure

\footnotetext{
*Corresponding author: a.k.chattopadhyay@ aston.ac.uk

${ }^{\dagger}$ tkkumar@gmail.com

${ }^{\ddagger}$ s.k.mallick@qmul.ac.uk
}

of poverty, we embed them within a broader community of individuals endowed with human skills as well as accumulated wealth and place them in an interacting market environment and a nonmarket state.

Our theoretical model assimilates ideas from an empirical law due to Engel, which says that consumption expenditure on any commodity is a function of income, together with another empirical observation made independently by Engel [1,2], Fox and Kumar [5], and Sitaramam et al. [6] (this was also established later by Choi and Moneta [7]) that there exists a hierarchical ordering of commodities and commodity groups according to a hierarchy of needs. We postulate that individuals adapt to the pattern of behavior of others in the community to which they belong. We use the observed long-term stationary pattern between consumption and income as the pattern that individuals adapt to. Thus, Engel curve plays a central role in our model describing the consumption behavior. We employ a specification of Engel curve that Kumar, Gore, and Sitaramam proposed [6] and later verified in Ref. [8]. These earlier works had shown that consumption deprivation of cereals (that is at the top of hierarchy of needs), defined as the shortfall of actual consumption from the saturation level, can be taken as a measure of poverty. However, those studies did not explain how income and consumption distributions arise, a key component of this article.

Our primary contribution here is the formulation and testing of an agent-based theoretical model of asset and commodity exchange in a market-based environment [9], and applying it to understand the resulting time varying income and consumption distributions. The best part of this exercise is that as a byproduct we arrive at a purely endogenous description and estimation of poverty as consumption deprivation of basic necessity (cereals, in the case of India). 
In our earlier studies on poverty (see Refs. [6] and [10]), the theoretical basis for the Engel curve remained unexplained. In this paper, we apply the same agent-based commodity exchange model that we developed elsewhere [7] as the basis for generating the Engel curve $[6,10]$. By juxtaposing the commodity and asset markets we are able to identify the basic defining feature of poverty as a level of income not adequate to compensate for consumption deprivation of a basic necessity. The most significant outcome of this marriage in concepts propounded in the older papers, with the new one, is that the characteristic features of poverty are endogenously determined by the nature of trade in an agent-based model. In particular, we find that the agents do not engage in trading of assets or even of luxuries as long as their basic necessities are not fulfilled, a feature emerging from the work by Engel, later studied by Kumar, Gore, and Sitaramam [6] and Choi and Moneta [7]. The poverty threshold thus emerges endogenously from the model as that level of income at which income is less than consumption deprivation of essential commodities. This is the direct link between poverty and consumption deprivation.

The plan of the rest of the paper is as follows. In Sec. II, we present our basic model that has two separate subsections, one for the Engel curve as a societal norm to emulate, a time-varying model for income distribution, and a time-varying model for consumption distribution, given the income. Section III describes the CD model and in this section, we also validate the models described in Sec. II. Section IV describes the data set we use and reviews the importance of alleviation of poverty as a pressing economic policy issue and how it was sidetracked with discussion on identifying the poor using poverty line instead of alleviating poverty. Applying our model, poverty or consumption deprivation is measured, monitored, and compared with regard to trends predicted from other measures of poverty. Section V summarizes our results and future directions.

\section{AN ECOBEHAVIORAL MODEL FOR DESCRIBING THE TIME-VARYING INCOME AND CONSUMPTION DISTRIBUTIONS}

\section{A. Engel curve as a societal norm for behavior}

The minimalist approach toward building an economic theory is based mainly on two premises: first, the economic agents exhibit their economic behavior in an environment that consists of their interaction with other economic agents, the institutions such as the markets, and the State; second, the agent's behavior is adaptive to reflect the basic traits of any living species to draw resources from that environment to survive and then to compete and succeed.

One of the guiding principles of economic behavior is to capture how to analyze class ascension with respect to income and consumption. Economic agents observe the profiles of income and consumption of others in their community and form an impression of the pattern of relationship between income and consumption. While each individual agent may have a subjective impression of what that pattern is, one can consider an average pattern. We tend to use the historical data on income and consumption expenditure and determine the steady-state equilibrium between the two and treat that as the

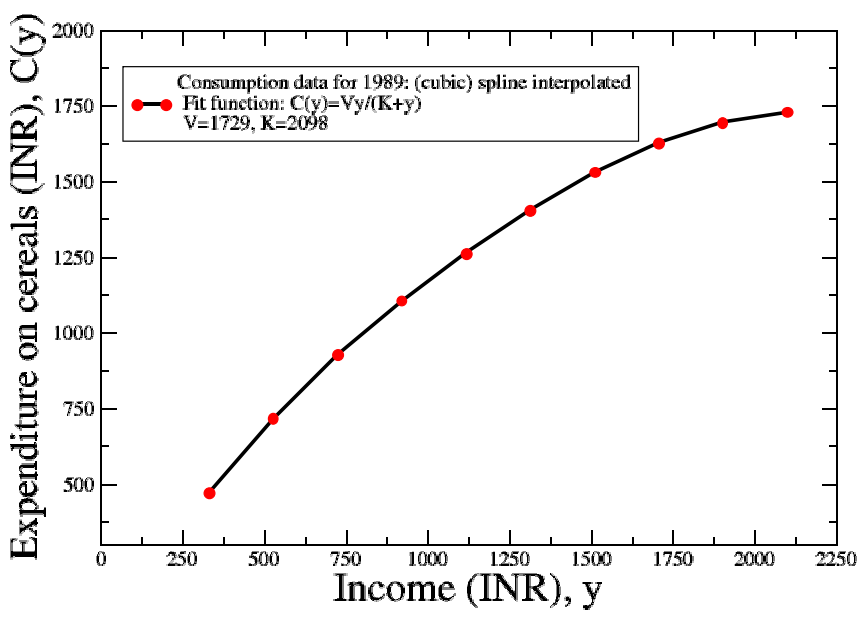

FIG. 1. A plot of the consumption function $C(y)$ against the expenditure $y$ on food grains. The data (dots) have been (cubic spline) fitted against the consumption function $C(y)=\frac{V y}{K+y}$ using a leastsquares method. To emphasize the saturation aspect corresponding to the given values of the parameters $(V=1729, K=2098)$, the $y$ and $C(y)$ variables have been, respectively, rescaled with $K$ and $V$. INR stands for Indian rupees.

norm that all economic agents follow. Engel [2] empirically derived a law showing that the proportion of total income spent on an essential item, food, is a decreasing function of income, or that the consumption of food as a function of income or total expenditure is a concave function. This is now widely known as Engel's law or Engel curve for essential commodities or necessities. While the nature of that law is very well-known, its mathematical form has been specified differently by different researchers. We had experimented with several such forms and found that a specification that biochemists normally use for saturation curves in bioassays conforms the best.

Correlating the NSS income data $y$ with that of the expenditure on basic cereals $C(y)$ [11], we find a good fit to a functional relationship [12]:

$$
C(y, t)=\frac{V(t) y}{K(t)+y},
$$

where the parameters $V(t)$ and $K(t)$ are time dependent $[8,12]$. $V(t)$ is a measure of the overall amount spent per family on cereal consumption when income is very large. This is the value of $C(y)$ in the plateau region of Fig. 1 for $y \rightarrow \infty$. $K(t)$, on the other hand, can be taken as the income necessary to support a consumption expenditure that is half of the maximum overall expenditure per family $[V(t)]$.

Incidentally, $K$ is also that level of income where consumption deprivation function and the affluence function (the actual consumption) will be equal. The consumption deprivation relates to the actual shortfall in the cereal expenditure from the maximum possible lifestyle based on cereal consumption alone and is defined as

$$
\mathrm{CD}[y(t)]=V(t)-C[y(t)]=\frac{V(t) K(t)}{K(t)+y(t)} .
$$

In our previous work [9], the parameters $V(t)$ and $K(t)$ were fixed from data that were available on a year-by-year basis from the World Bank repository [11]. For the same reason, though, 
the analysis was limited to the plausible range of data without any predictive power for future times for which data is not available. The only way to ingrain a generic time dependence in the consumption function that can probabilistically predict values of $C(y)$ for future times is to have a time evolving model for $C[y(t)]$ itself, which is the main theme of this paper.

\section{B. Time-varying income distribution}

We now present a model for the evolution of income distribution over time. This income is generated from the underlying market exchange for assets, including human capital that produces labor services as a flow emanating from that stock of human capital. However, there are two kinds of economic agents, one kind that have only their own labor skills that fetch them only a low level of income not even adequate to meet their basic necessities and thus incur a consumption deprivation. This deprivation is defined as the shortfall of consumption from the saturation level (maximum consumption at any level of income of an essential commodity or basic need). The other kind are those whose income is more than the consumption deprivation and thus engage in the asset market exchange.

The agents (people) are motivated to improve their level of living by moving up the income ladder. This is done through an adaptive mechanism with maximum mean income as the goal to seek, each year a fraction of that being added to their income. However, when people save out of the income over and above the consumption deprivation, a fraction of that saving is subtracted from the income for that period. Apart from these two factors that determine the rate of growth of income, there could be other stochastic disturbances, the magnitude of which might depend on the level of income; the greater the income, the greater is the variability in income, as people put their incomes in increasingly risky portfolios.

As detailed in our earlier studies [6,10,12], our choice for this minimum of income threshold for participation in the asset market is the consumption deprivation function $\mathrm{CD}\left(y_{i}, t\right)$, which is the amount of income lacking in an income class $i$ that is needed to reach the saturation level of consumption of the essential commodity (cereal, in our case). This threshold is a function of the income $y_{i}(t)$ at time $t$. While the increase in the spending power of the entire population is a motivating factor and a goal for growth in income, a transfer of wealth (e.g., investment by agent $i$ through trade) will result in decreased spending power for this agent that will oppose the positive growth rate defined by $\bar{Y}_{i}(t)$. What this implies is that a household will not have any savings in assets as long as the income is less than the consumption deprivation. This income-expenditure balance is further driven by ambient market economics that may randomly aid or deplete the trading wealth flow, necessitating the presence of stochastic term in the dynamics.

The resultant stochastic (Langevin) model that we then get from this construction is as follows:

$$
\begin{aligned}
\frac{d y_{i}}{d t} & =\beta \bar{Y}_{i}(t)-\alpha\left(y_{i}-\mathrm{CD}_{i}\right)+\eta_{i}(t) y_{i}, \\
\left\langle\eta_{i}(t) \eta_{j}\left(t^{\prime}\right)\right\rangle & =D_{0} \delta\left(t-t^{\prime}\right) \delta_{i j},
\end{aligned}
$$

where $\beta \bar{Y}_{i}(t)$ is the maximum level of income (proportional to the mean growth rate $\bar{Y}_{i}(t)$ of income) at time $t$ for the $i$ th class of individuals that they would like to aim at but achieve only a fraction of it in each period. This is a sum of desired consumption expenditure and desired savings. This is thus partly an adaptive behavior, the first term being a motivational force that creates incentives to increase the level of income, and the second term, on the other hand, defines how increase in income is offset by a decrease in income available for consumption in the current period (due to savings). It assumes that a proportion $\alpha$ of income is saved and is not available for spending in the current period. $\eta_{i}(t)$ is the previously alluded stochastic factor generated by the uncertain market environment; the greater the value of income and trade, the larger is the stochastic disturbance to modulate the income.

As is customary in finance literature [13], the model uses a multiplicative noise structure based on Ito calculus to portray the Markovian nature of the inherent time dynamics. Conceptually, this is a preferred choice than a Stratonovichtype structure (these two approaches are interconvertible using a transformation) because while integrating through the time domain, the left endpoints of the intervals in the limiting process could be chosen which is more conducive to finance as we do not know the future stock prices. In the Stratonovich calculus each time step is the midpoint between the previous step and the present step. Thus, the Ito approach preserves the "martingale property," while the Stratonovich approach does not. The contribution coming from the market uncertainty in our model depends on the choice of the distribution of the stochastic term [14]. In the context of the present model, a multiplicative noise structure will mean that uncertainty associated with the retention of large wealth is high and vice versa.

In line with the approach taken in our earlier papers [9] and [12], we analyze the equilibrium income distribution first, from which information about the time dynamics is extracted, using a year by year evolution of this income probability density function (PDF). In this paper, we take that solution as given and examine the commodity exchange market the same way as we examined the asset market equilibrium. The basic idea of trading of commodities for consumption between agents remains more or less the same as in Ref. [9]: an agent $i$ can spend an amount $y_{i}$ while being subjected to stochastic effects of trading $\eta_{i}(t)$ at time $t$. The rate of increase of the agent's ability to spend more money on a commodity will be principally determined by two factors, the (time-dependent) maximum consumption expenditure on essential commodities by the entire reference population to which the household belongs, $V(t)$, and total income available for consumption expenditure as derived from the asset market distribution. We are thus assuming a recursive behavior, making a decision on work, income, and savings first and then using the income available for consumption to make consumption expenditure decisions.

The above form of the stochastic income-expenditure growth rate above the threshold line $\operatorname{CD}_{i}(y, t)$ leads to the following Fokker-Planck equation [36], which depicts the time rate of change of the income distribution function:

$$
\frac{\partial \hat{f}}{\partial t}(y, t)=\frac{\partial}{\partial y}\left\{[(\alpha+2) y-C(t)-\operatorname{CD}(y, t)] \hat{f}+y^{2} \frac{\partial \hat{f}}{\partial y}\right\} .
$$


The coupled dynamics described above, involving the probability density function of income $f(y, t)$ and consumption deprivation function $\mathrm{CD}(y, t)$, represents the fact that effective trade in assets ensues only when $y>C D$, as discussed before.

In the steady state $\bar{C}(t)=C_{0}$ and $\operatorname{CD}(y)=\frac{V_{0} K_{0}}{K_{0}+y}(t \rightarrow \infty$ limit), which gives us the steady-state income distribution:

$$
\hat{f}(y)_{t \rightarrow \infty} \propto \frac{e^{-\frac{\left(C_{0}+V_{0}\right)}{y}}}{y^{\alpha+2}}\left(1+\frac{K_{0}}{y}\right)^{V_{0} / K_{0}} .
$$

Here $V_{0}$ and $K_{0}$ are the values of $V(t)$ and $K(t)$ for the corresponding year concerned. The proportionality constant can be evaluated from the condition $\int_{y_{0}}^{\infty} \hat{f}(y)_{t \rightarrow \infty} d y=1$.

The parameter $\alpha$ varies from economy to economy and can be evaluated from data. For our study, we use the Indian data recorded in the Indian National Sample Survey (NSS) [11] spanning 43 years (1959-2002) across 25 surveys and sampling about 7 million expenditure (a proxy for income) data. Only food consumption statistics has been used, that being the modicum of basic life disbursement for the lowest income sectors (destitute). The monthly income and expense data are made available across various income sectors, popularly known as "expenditure classes," using which the cumulative distribution function (CDF) was plotted. The CDF led to the probability density function (PDF). For the purpose of this analysis, all data have been deflated using the consumer price index (CPI) data (also available from Ref. [11]), the conversion formula being

$$
\text { Deflated expenditure }=\frac{\text { Raw expenditure data }}{\text { CPI }} .
$$

This empirical relation between cereal consumption expenditure and total expenditure (taken as a proxy for income) has been previously established by us using the same NSSO data for several rounds of NSSO and for several Indian states and for all of India (see Ref. [8]). Having validated our sub-model A, we next consider the time dynamics model (sub-model B) for income using Eq. (5). Using the NSSO data for 1974, 1983, and 1991, we estimated the CDF and the corresponding density functions and plotted them against the values simulated by our model. The results are depicted in Fig. 2. The inset shows that the IPDF emerging from our model is also in excellent agreement with the functional form provided in Eq. (5).

As shown in Ref. [9], Eq. (4) can be analytically solved to obtain the full time-dependent solution as a sum of confluent hypergeometric functions $F(a, b, z)$ with time-dependent coefficients:

$$
\hat{f}(y, t)=\sum_{n=0}^{n=\infty} \exp \left(-\omega_{n} t\right) g_{n}(y),
$$

where $\omega_{n}=2 \pi n$ and

$$
\begin{aligned}
g_{n}(y)= & B_{1}\left[\frac{c(t)}{y}\right]^{\gamma_{-}^{(1)}} F\left[\gamma_{-}^{(1)}, \gamma_{-}^{(2)},-\frac{C(t)}{y}\right] \\
& +B_{2}\left[\frac{C(t)}{y}\right]^{\gamma_{+}^{(1)}} F\left[\gamma_{+}^{(1)}, \gamma_{+}^{(2)},-\frac{c(t)}{y}\right],
\end{aligned}
$$

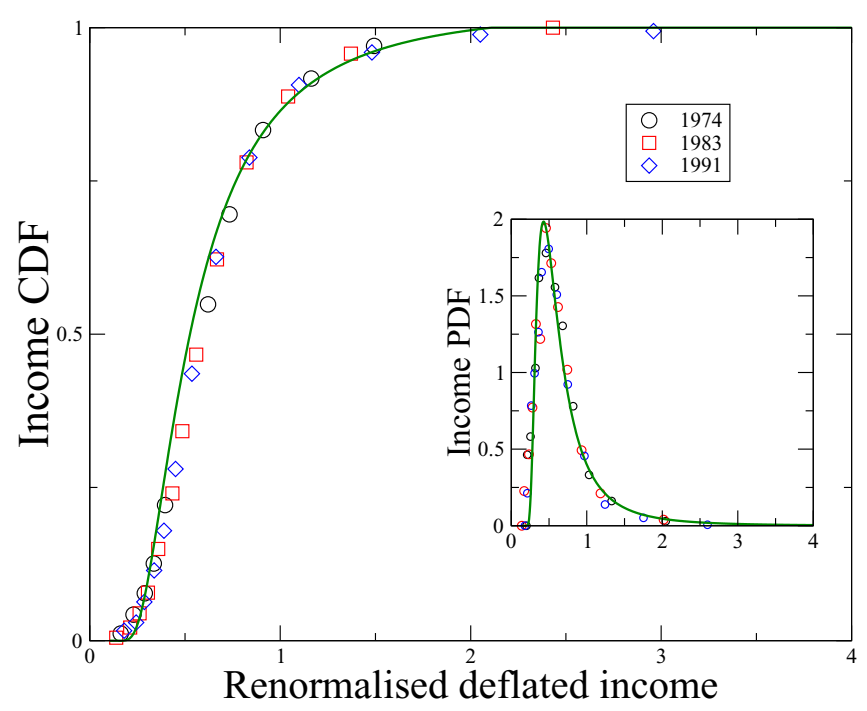

FIG. 2. Plots of the cumulative distribution functions (CDFs) against deflated income for selected years, with inflation independently sourced from the consumer price index (CPI) and renormalized to the 1974 mean income in rupees (64.84 INR). The green line is our theoretical curve, taking $y_{i}$ as income above a nonzero level below which agents would die of starvation (set at 0.15 in renormalized units). Inset shows the IPDF, which is the differential of the CDF, evaluated from the data by interpolation. The points are the real data from NSS, the line is our analytic function for the steady-state distribution that fits with the power-law predicted in Eq. (5) with $\alpha=1.6$.

$$
\begin{gathered}
\gamma_{ \pm}^{(1)}=\frac{3+\alpha \pm \sqrt{(1+\alpha)^{2}+4 \omega_{n}}}{2}, \\
\gamma_{ \pm}^{(2)}=1 \pm \sqrt{(1+\alpha)^{2}+4 \omega_{n}},
\end{gathered}
$$

and $B_{1}$ and $B_{2}$ are constants dependent on initial conditions. For the Indian dataset used, the adjustable (changing with the economy) parameter $\alpha=1.6$ confirms fractional Brownian motion for the stochastic model [15].

\section{THE CONSUMPTION-DEPRIVATION DYNAMICS}

Given initial income, initial consumption of cereals is determined as per the societal consumption pattern as depicted in the Engel curve. This is followed by an estimation of the consumption-deprivation abiding Eq. (2). Then the next period income is generated from a solution of the Fokker-Planck equation described in sub-model $\mathrm{B}$ above. Consumption for the next period is determined, given initial income and consumption and the societal pattern of consumption behavior, from the consumption deprivation model presented below.

In the following, we demonstrate a phenomenological derivation of the "consumption deprivation" (CD) kinetics of agent $i$ who has income $y$ line at time $t$. This income is still deficient by $\mathrm{CD}(y, t)$ in restoring the "consumption" function back to the saturation level $V(t)$. The question then would be the dynamical "instability" that may be created due to influx (or outflux) of wealth fraction $\Delta y$ to (or from) agent $i$. Surely, such a change will initiate a competition of two opposing forces of 


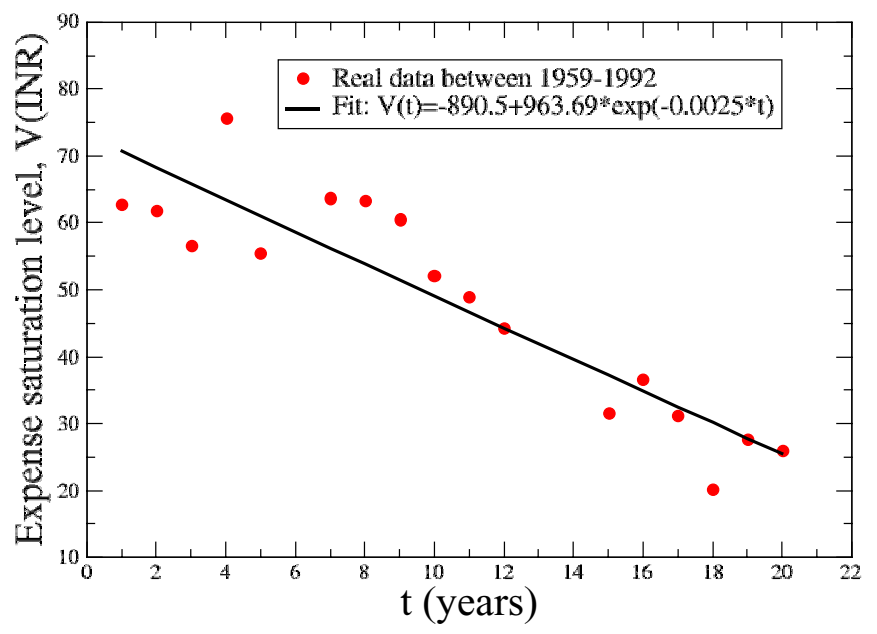

FIG. 3. $V(t)$ versus $t ; V(t)=-890.5+963.69 * \exp (-0.0025 * t)$ for $V(t)>0$. The dots represent real data points while the solid line is the least-square fitted trendline through these points between $1960<t<1992$.

economics: one that will try to "neutralize" the effects of this spike in deprivation through uniform homogenization of assets across all wealth states characterized by $y$ and the other that will strive to work against this effect by maximizing a "lateral growth" leading to wealth piling in nearest income states $y \pm \Delta y$; in other words, additional income will just be saved without explicitly affecting the consumption expenditure. The first effect can be easily represented by a Laplacian diffusion term (not in money, but in CD) $v(t) \frac{\partial^{2}}{\partial y^{2}} \mathrm{CD}(y, t)(v(t)$ : time dependent diffusion constant) while the latter term depends both on the transient poverty $\operatorname{CD}(y, t)$ itself, together with that of the gradient of the poverty increase $\frac{\partial}{\partial y} \operatorname{CD}(y, t)$ across neighboring wealth sectors $[y-\Delta y, y+\Delta y]$; in other words, on the product $\mathrm{CD}(y, t) \frac{\partial}{\partial y} \mathrm{CD}(y, t)$.

This second (reflection) symmetry violating term implies that the poorest people $(y \rightarrow 0)$ achieve the fastest poverty reduction, although, such a scheme does not allow for conservation of income as is reasonable. $V$ can be increased either by an increase in mean income and a decrease in inequality of income (uniform homogenization) or increase in mean income with an increase in inequality of income (lateral growth with wealth piling). Likewise, $K$ can be increased or decreased affecting the poor and rich differently where the prices are changed differently, like through the public distribution system of foodgrains through the fairprice shops.

Combining both terms, respectively, representing economic neutralization around the saturation level $V(t)$ (diffusion dynamics) that is perturbed by a directed economic gradient between neighboring agents (Burgers' nonlinearity [16]), we arrive at our time dynamical model for consumption deprivation:

$$
\begin{aligned}
& \frac{\partial}{\partial t} \mathrm{CD}(y, t)+\mathrm{CD}(y, t) \frac{\partial}{\partial y} \operatorname{CD}(y, t)=v(t) \frac{\partial^{2}}{\partial y^{2}} \mathrm{CD}(y, t) \\
& \frac{\partial}{\partial t} \mathrm{CD}(y, t)=V(t) K(t) \frac{2 v(t)+V(t) K(t)}{(K(t)+y)^{3}} .
\end{aligned}
$$

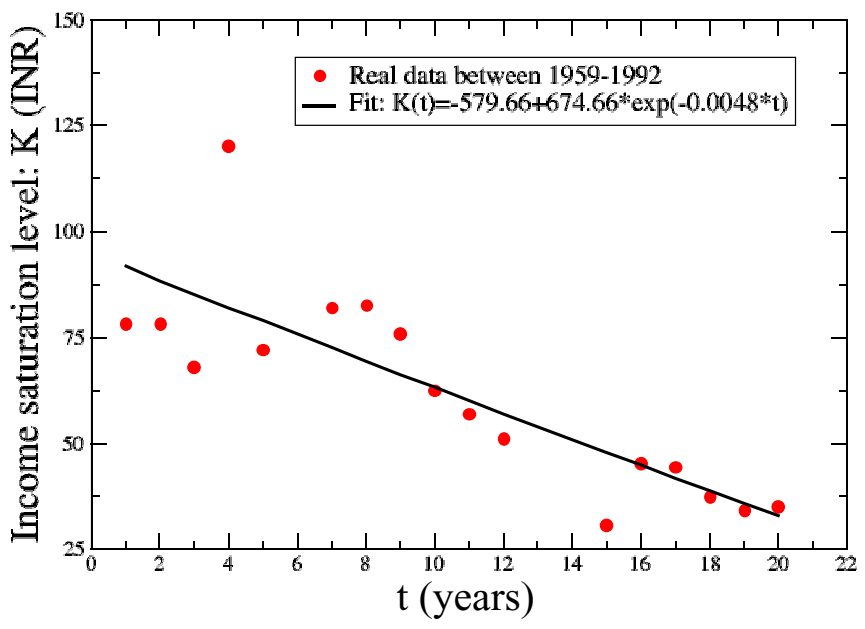

FIG. 4. $K(t)$ versus $t ; K(t)=-579.66+674.66 * \exp (-0.0048 * t)$ for $K(t)>0$. The dots represent real data points while the solid line is the least-square fitted trendline through these points between $1960<t<1992$.

In both Figs. 3 and 4, the $x$ axes use "round numbers" depicting timelines, rather than year numbers; this is to allay the aperiodic nature of data collection over the years.

The increase (or decrease) in poverty is then simply related to whether $\Phi(t)=2 v(t)+V(t) K(t)>0$ (or $\Phi(t)<0$ for poverty decrease).

In order to stipulate a value, or at least a regime, for $v(t)$, we need to estimate the stationary state statistics of our dynamical model with that from our previous data-based studies $[9,12]$. As simple algebra will suggest, a stationarystate solution $\left(\frac{\partial}{\partial t} \mathrm{CD}(y, t)=0\right)$ of our model gives $\operatorname{CD}(y, t=$ $\left.t_{0}\right)=\frac{-2 v_{0}}{K_{0}+y}$, where $K_{0}$ is the value of the parameter $K(t)$ at $t=t_{0}$. A comparison of this steady-state solution with that from Ref. [12] will further ensure that $v_{0}=-\frac{V_{0} K_{0}}{2}$, thereby nonequivocally establishing the steady-state form of $v(t)$. For the time dynamical evolution of the CD function, we will assume a solution that is not too far from the linearly stabilized steady state defined by $v_{0}$, i.e., $v(t)=-\frac{V_{0} K_{0}}{2}+\delta v(t)$, ensuring time evolution of the CD function by maintaining $\Phi(t) \neq 0$, where $\delta v(t)$ quantifies the nonequilibrium increment in $v(t)$ as a function of time.

This can be done without any loss of generality since our actual data analysis from Ref. [9] has already ensured that PDF statistics from each yearly data matches with the corresponding steady-state solution of the original Fokker-Planck model given in Eq. (4). The choice of such a hydrodynamic model also ensures the implicit presence of long-ranged "hydrodynamic interactions," which are believed to be so vital in understanding financial peaks and troughs from agent-based modeling studies [13].

\section{RESULTS AND DISCUSSIONS}

We cross-validate our model described in the previous section (data capture) with data from India for several years. These are the same data that were extensively used by several researchers on economics of poverty. Deaton also used this data [3] on household consumer expenditure in India obtained 


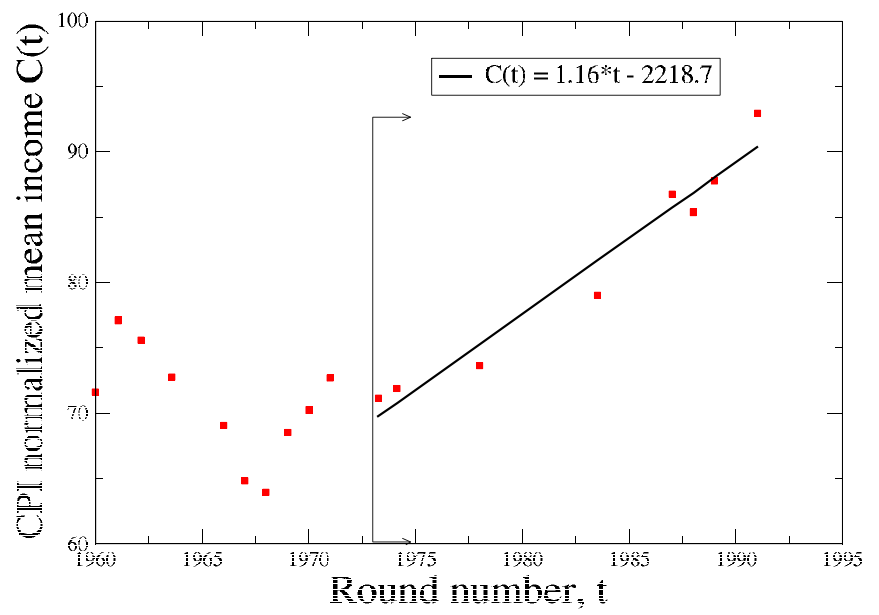

FIG. 5. CPI normalized mean income plotted against time. There is a monotonically increasing trend post 1973 (indicated by a marker in the figure), the year all poverty data got standardized, a behavior that is compatible with real expectations. The corresponding leastsquare regression fit is estimated as $C(t)=1.16 t-2218.7$ between $1973<t<1992$.

by the National Sample Survey Organization (NSSO) over several years, quite often conducted every five years with very large sample size and more frequently in years in between with a much smaller sample size. The data have been archived and made available through the World Bank data depository [11], which we used. Using the consumption expenditure data for the year 1989, we observe the following data fit (Fig. 1) for the Engel curve specified in Eq. (1) above in our sub-model A. Mean income in India has improved since 1973 when the poverty estimates were standardized and revised. Figure 5 plots the mean income in India after adjusting for price variation.

Using Cole-Hopf transformation [16], the closed-form time-dependent solution of the consumption deprivation function for constant parameter values $V_{0}$ and $K_{0}$ from Eq. (11) can be written as

$$
\begin{aligned}
\mathrm{CD}(y, t)= & -2 v_{0} \frac{\partial}{\partial y} \log \left[\left(4 \pi v_{0} t\right)^{-1 / 2} \int_{y_{0}}^{\infty} d y_{1} e^{-\frac{\left(y-y_{1}\right)^{2}}{4 v_{0} t}}\right. \\
& \left.\times e^{-\frac{1}{2 v_{0}} \int_{0}^{y_{1}} d y_{2} \operatorname{CD}\left(y_{2}, 0\right)}\right]
\end{aligned}
$$

where $\mathrm{CD}(y, 0)$ is the initial value of the function $\operatorname{CD}(y, t)$. This solution assumes a fixed value of the parameters $V$ and $K$ for any year as its initial condition and then uses that to arrive at $C D$ values for future times as given in Eq. (12). In other words, Eq. (12) requires the initial values of $V$ and $K$ at some initial time $t_{0}$ as its initial condition that could be drawn from the steady-state distributions, respectively, of $C(y)$ [from Eq. (11)] and $y$ [from Eq. (5)] to be probabilistically accurate. At the level of linear stability analysis, this means that one does not even need to have a priori knowledge of the nature of time dependence of the variables $V(t)$ and $K(t)$, which makes the description properly probabilistic. The implication of such time-evolving consumption-deprivation dynamic formulation of inequality measure cannot be overstated since such a structure will self-consistently relate to appropriate risk analysis and related derivative models, an aspect that is largely believed to have been responsible for the systemic failure of economic theories in predicting the last few rounds of financial crisis [17].

\section{A. Application of the model to estimate poverty in India}

The main focus of this article is to establish the usefulness of our theoretical model to define and estimate poverty, expatiating an altogether novel and much more meaningful way than how the subject has been handled by economists so far. The history of poverty studies shows that Rowntree [19] used a poverty line of income to define who the poor were. Later Sen [18] used Rowntree's [19] concept of identifying the poor using his axiomatic approach by relying on Dalton and Pigou's work [20] on income inequality and economic welfare on the one hand and the effect of tax and subsidies on welfare, but applied only for analyzing income inequality amongst the poor. Follow-up works [21-24] followed similar lines using exogenous poverty line and income distributions. While data-based inequality studies [25] extend beyond the scope of developing countries, all such approaches have relied on an exogenous reference to poverty line. The nature of such constrained studies could be easily demonstrated using test cases, an example of which is the axiomatic analogy used by Sen that focused explicitly on a poverty line of income and related income gap measures of poverty. But this approach, as like many others, did not relate poverty measures to what one really means by poverty and hence alienated themselves from the real concept of poverty. With their preoccupation of defining who the poor are, the definition of poverty as a fundamental expression of consumption deprivation increasingly got lost in jargons.

While Shorrocks [26] and Kumar et al. [10] pointed out the weakness of a thin dividing line to delineate the poor from the nonpoor, our approach toward poverty measurement presented here avoids a discontinuous treatment of the poor and nonpoor and poverty by using a detailed and disaggregated agent-based model to discover those economic agents whose initial endowment of (mostly human) assets (e.g., education and skills) is such that they continue to remain within a band of high consumption deprivation most of the time, a situation highlighted by Carter and Barrett [24] and Baulch and Hoddinott [27]. As one can see, our model is offering an entirely different perspective of poverty defined from income and consumption expenditure, which is very different compared to traditional economics approach. As Colander observed [17], one of the reasons for the failure of economic models to predict the financial crisis in 2008 was a systemic failure by economists not to entertain models outside a narrow range of mainstream models. We believe that our model offers not only a viable alternative but also opens up better tools for the analysis and policy related to poverty and poverty alleviation.

Both parameters $V(t)$ and $K(t)$ are known to show strong time variations (as shown in Figs. 3 and 4); this is a very suggestive trend and needs to be incorporated in all analyses. Figure 5 shows the variation of (CPI normalized) mean income over years. The fast rising mean income in the post-1973 era, as evident from these Indian data, is commensurate with the improving economic situation of the country and is a 


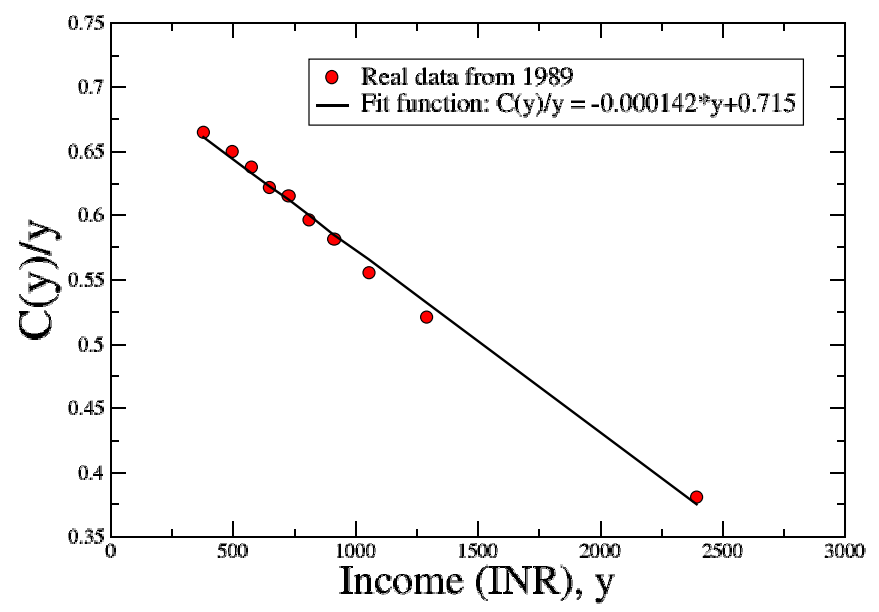

FIG. 6. A plot of the ratio of the consumption function to the income $C(y) / y$ that is seen to follow a linear decaying trend with $y$ (data as in Fig. 1).

complementary description to decreasing poverty trends, as could be seen from Figs. 3 and 4, respectively. Figure 6 reminds the Engel prediction [2] that as $y$ increases, the $C(y) / y$ fraction linearly converges to a very low value (data from 1989, as in Fig. 1).

In the following, we solve the dynamical system of Eqs. (4), (11), and (13) numerically for the initial value $\operatorname{CD}\left(y_{0}\right)=$ $\frac{V_{0} K_{0}}{K_{0}+y_{0}} \approx \frac{73.19 \times 95}{95+30}=55.62$ using nonlinear regression fits from real data $(V(t)=-890.5+963.69 \exp (-0.0025 t)$ and $K(t)=-579.66+674.66 \exp (-0.0048 t) ; \quad V(t), K(t)>0)$ as shown in Figs. 3 and 4 to obtain the time series estimate of poverty (Fig. 7). In the limit of destitution $(y \rightarrow 0)$, one can easily see that $C(y) / y(t) \rightarrow \frac{V(t)}{K(t)}$, that is the budget share of essential commodities. The quantity $V_{0} / K_{0}(=0.77)$ is the budget share of the poor. The proposed formulation of the CD dynamics thus has far-reaching consequences, in that it reconfirms the Engel prediction in the steady-state

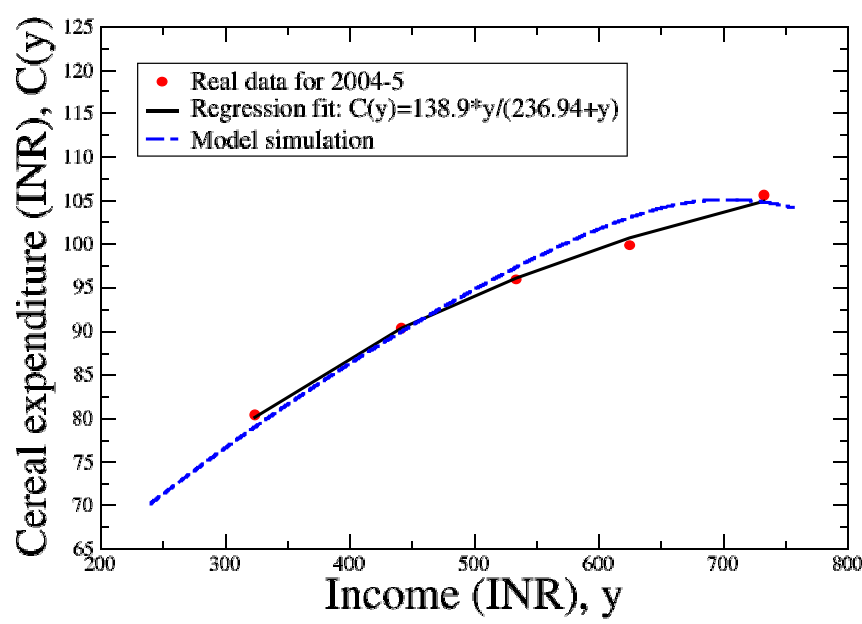

FIG. 7. Plots comparing cereal expenditure versus income (all in Indian rupees: INR) data for the year 2004-2005 [28] (solid circles) with the Engel form fit (solid straight line) and the numerical solution (dots) of Eq. (11). limit $\left(\frac{\partial}{\partial t} \mathrm{CD}=0\right)$, the fact that $C_{\text {steady state }}(y)=\frac{V_{0} y}{K_{0}+y}$, where $V_{0}(=73.19)$ and $K_{0}(=95)$ are the fixed point values of $V(t)$ and $K(t)$, respectively. The "theoretical turnover time" defining the validity of the nonlinear regression fits of $V(t)$ and $K(t)$ are accurate up to about 31.5 rounds, which in year numbers is roughly equal to 2005, as shown in Fig. 7. The model is not restricted to these numbers, though, since a running average can be continuously done to stretch the range of validity to whatever timeline is required.

It is clear from Eq. (1) that as $y \rightarrow 0, C(y) / y \propto V(t) / K(t)$. Thus the steady-state solutions for $V(t)$ and $K(t)$ and $V(t) / K(t)$ have a significant policy implication in line with what Engel had originally hypothesized. That ratio represents the share of the expenditure on the basic necessity in total income or total expenditure. Figure 6 below reproduces the model-predicted $C(y) / y$. The observed pattern of this proportion of income spent on food as a function of income is just as suggested by Engel. Summing up, the performance of our model is presented below in Fig. 7 with the actual data on cereal consumption expenditure and total expenditure, the fitted Engel curve, and the model simulated values for the year 2004-2005. Some further explanation of the simulated solution is provided below.

The fast rising mean income in the price-adjusted post-1973 era (Fig. 5), as evident from these Indian data, is commensurate with the improving economic situation of the country and is a complementary description to decreasing poverty trends, as could be seen from Figs. 3 and 4, respectively.

The dotted points in Fig. 6 are the actual data points that are compared against a regression fit straight line. This linear plot reminds one of the Engel prediction [2], which originally explained why food-related expenditure can only follow a "monotonically decaying" profile with increasing income. The statistics in Fig. 7 complements this description.

\section{B. The "endogenous" poverty index}

As a major confirmation of the hypothesis used to track the CD dynamics, Fig. 7 shows a comparison of the actual model data [28] (dotted line) for the year 2004-2005 with the Engel curve (solid line) hypothesis and, thereafter, an income:expenditure analyzed from a solution of Eq. (11). The simulation here uses the deterministic limit of Eq. (2), in that this leads to an average income that can also be calculated from Eq. (3), from a knowledge of the probability density function in Eq. (5), using the relation $\langle y\rangle=\int_{y_{0}}^{\infty} d y$ y $\hat{f}(y, t)$; here the angle brackets " \langle\rangle " represent ensemble average. In this estimation, we have used the functional representation of $V(t)$ and $K(t)$ as shown in Figs. 3 and 4. The $y$ shown in this figure is actually this ensemble averaged $\langle y\rangle$ and the corresponding consumption function is $C(\langle y\rangle)$; for brevity as also for the sake of our general line of reasoning, we have dropped the angle brackets " \langle\rangle " in the plot. The quality of Engel's predictions and the strength of our complementary model can be justified both from the fit (solid line against real data circles) as well as from the solution (dotted line) of our proposed model represented in Eq. (11).

As always, the strength of an assumption can only be proved from the efficacy of its quantitative measurable output, which, in our case, will be the "poverty index." Following 
Refs. $[9,10,29,30]$, the poverty index $P_{\mathrm{CD}}(t)$ is defined as the statistical average of the consumption deprivation function across the entire range of income:

$$
P_{\mathrm{CD}}(t)=\int_{y_{0}}^{\infty} d y \operatorname{CD}(y, t) \hat{f}(y, t),
$$

where $\operatorname{CD}(y, t)$ and $\hat{f}(y, t)$ will be, respectively, obtained from Eqs. (11) and (4). The time varying mean income $C(t)$ present in Eq. (11) can be evaluated from the relation $C(t)=$ $\int_{y_{0}}^{\infty} d y y f(y, t)$, which addresses the oscillatory instability that would otherwise destabilize the numerical simulation, should the $C(t)=1.16 t-2218.7$ fit function be used instead.

Remarkably, from our (deflated) NSS data over 23 years, it can be seen from Figs. 3 and 4 that both parameters admit of approximate linear regression fits with time-decaying trends. In the post-1973 regime, when all poverty data were renormalized for the first time, the mean income profile too follows an upward linear trend as shown in Fig. 5 that fits well with the decaying linear trends depicted in the $V(t)$ and $K(t)$ regression fits. The legends to these figures show the relevant extrapolation formulas $(V, K>0, \forall t)$. Apart from clearly indicating a (linear) trend in the deflated time series statistics, the starting points (round 6) of both these plots define the initial condition $\operatorname{CD}(y, 0)$ that is needed to solve Eq. (4). The progressively diminishing values both for $V(t)$ and $K(t)$ are not so difficult to anticipate, since major economic decisions during these periods contributed in major economic improvement of the society, a fact that also tallies with conventional index-based analyses. It must be noted, though, that such fits are limited to positive definite values of $V$ and $K$ only.

As a cross-check of the strength of our theory, we compared our new theoretical poverty index with data from all three indices popularly used in the literature: the head count (HI) index, the poverty gap (PG) index, and the squared poverty gap (SPG) index. Additionally, we also compared this with a similar statistics obtained from our previous work (Fig. 4 in Ref. [9]).

The result (Fig. 8) shows good qualitative agreement between the new CD dynamics modified index with all three indices compared to that in Ref. [12], a plot that is represented in Fig. 8 as "CD-fit theory." While the new time dynamics adjusted poverty index does not improve the quantitative result compared to the poverty index calculated earlier [9], its strength lies in its probabilistic prediction ability as is demonstrated in the values beyond 1992. An important stabilizing feature of this mechanism is that the evaluation of this new CD-dependent poverty index does not rely on any regression fitting of the time evolution of the mean income of the population. This arguably edges out the occasional hikes (as in 1967) and dips (as in 1988), but at a statistical level, this gives quite reliable estimates of the poverty index for future times. The strong upward trend shown in the mean income variation with time (Fig. 5) is ingrained in this analysis through the usage of the ensemble averaged definition $C(t)=\int_{y_{0}}^{\infty} y \hat{f}(y, t) d y$ and should be the definition used; this obfuscates the strong oscillations shown in the growth profile of $C(t)$. In our simulations, we considered $y_{0}=30$

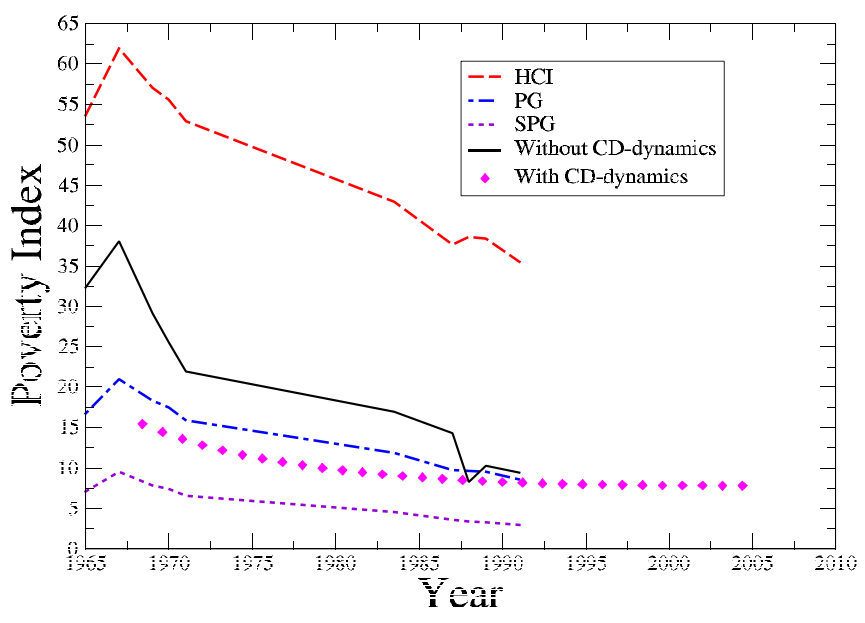

FIG. 8. Plots of the different poverty indices-head-count index (HCI, dashed); poverty gap (PG, dot-dashed) index; squared poverty gap (SPG, dotted) index - against the previous [9] work ("without CD dynamics," solid line) and that against the new CD dynamics ("with CD dynamics," diamonds) adjusted estimation. Post-1965, the mean profile of the CD dynamics modified theoretical index is shown to be in a steady decline across the years. The new theoretical index has also the major advantage of being able to forecast future poverty line statistics based on linear regression fits as depicted in Figs. 3 and 4. The part beyond 1992 shows the prediction made from this theory that can be tested against real data.

(CPI normalized income in year 1965) as the CPI adjusted per capita daily income.

In order to cross-check the veracity of this modified poverty index, we used HCI data from Ref. [11] for the years 2005 and 2010, together with relevant CPI values from the Reserve Bank of India [48] for these two years. The relative HCI indices for these two years (37.2\% in 2005 versus $29.8 \%$ in 2010 ) scale identically as the new model index (2.16 units in 2005 against 1.73 units in 2010) to within $95 \%$ accuracy.

The combination of results from a numerical solution of Eq. (11) as shown in Fig. 7, and the Engel fit form $C(y)=\frac{V y}{K+y}$ for 2004-2005 data [28] proves that the corresponding income needed to consume an amount $V / 2(=69.5)$ is matched by a corresponding saturation income of $y=K(=236.94)$. In other words, our model is robust enough to forecast an effective "steady state" in the poverty dynamics of the Indian economy at around 2004-2005 that shows up as a plateau in the PI time profile. As our model is an adaptive model of the Markovian type, the model predictions are a period ahead. Based on quinquennial surveys this means that we can predict the level of poverty five years hence. Note that while the extrapolation-based results for the parameters $V(t)$ and $K(t)$ are constrained to the immediate vicinity of the linearly stable regime, the feedback architecture inlaid into the present structure allows for repetitive running averages over immediate neighborhoods leading to an extended time regime of prediction. We are extremely encouraged by the success of our model in capturing the real data the way it did. It will be our endeavor to improve the model even further in future to improve the data capture.

It is clear from this diagram that our earlier model with income dynamics tracked the kinks in the traditional poverty 
indices quite well, whereas the CD dynamics version (the present version) did not. This only suggests that our earlier model, based on time-varying income, predicted income distribution well and hence predicted income-based poverty index well. Our present model, being based in addition on $\mathrm{CD}$ dynamics, is possibly tracking a consumption-based consumption-deprivation index better than poverty indices based on income. This is a topic we shall examine in greater detail in our next research endeavor.

\section{CONCLUSIONS}

Our study here addresses poverty measure head-on by defining what poverty is as a measure of consumption deprivation. Using a microeconomic theory of agent-based adaptive behavior, where people look at the society at large $[31,32]$ and their income and consumption pattern (data driven approach to adaptive behavior), both within and outside their own income classes, and adapt their consumption to the societal consumption pattern, our model structures a selfconsistent endogenous structure (based on consumption statistics), instead of an axiomatic model reliant on an exogenous poverty line that remains subjective and exogenous largely uncontrolled by evidence-based data. Starting from an agentbased economic exchange model, the relevant time-dependent income distribution is first derived in this article; in parallel, the time evolution of the consumption-deprivation dynamics too is modeled homogeneously without any exogenous parameter like a poverty line. The combination of these two dynamics leads to a self-sufficient inequality measure that can be probabilistically extended to predict the future inequality landscape.

The result demonstrated in Fig. 8 shows decent agreement between the new CD-dynamics modified index with the HCI, PG, and SPG indices, together with the theoretical index (constrained to data only) previously estimated in Ref. [12], a plot that is represented in Fig. 8 as "CD-fit theory." While the new time dynamics adjusted poverty index does not improve the quantitative result compared to the poverty index calculated earlier [9] and hence is a "null result" from that context, the uniqueness of this new study is in its ability to predict the future indices. The fact that parameters $V(t)$ and $K(t)$ are now regressively linked to the core dynamics of the poverty evolution, this new model allows for probabilistic future predictions. In order to cross-check the veracity of this modified poverty index, we used HCI data from Ref. [11] for the years 2005 and 2010, together with relevant CPI values from the Reserve Bank of India [48] for these two years. The relative HCI indices for these two years (37.2\% in 2005 versus $29.8 \%$ in 2010 ) scale identically as the new model index (2.16 units in 2005 against 1.73 units in 2010) to within $95 \%$ accuracy. At this point, it is worthwhile to remember that since we are rejecting the focus axiom of Sen (i.e., the poverty line) [18], strictly speaking, there is no point in validating our results with HCI and PGI that depend on that axiom. But as both indices track consumption deprivation, one with a poverty line and the other without (in the traditional poverty economics these indices are referred to as exclusive and inclusive indices), we only check for the direction of trend and not the actual magnitude.
Such basic success in probabilistic prediction of the gross qualitative aspects of the poverty index evolution emphasizes the need for advancing more accurate models, which are also based on more elaborate multivariate modeling [33,34] and data analysis [35] that will go beyond the present restrictions through more accurate prediction of the primary agent-based Langevin model [36], Eq. (3a), and thereafter integrating the same with the presently espoused consumption deprivation dynamics. The dynamical stochastic Eq. (3a) also gives some indication of the effect of redistributed income between expenditure classes on overall mean income. Typically we find that such redistribution reduces poverty during the period of application but suppresses the mean income, such that if the redistribution is removed (or, in the long term, even if it remains) then the poverty may increase again, unless some external agent, such as improved technology, contributes in a still faster rate of increase of the mean income. A poignant feature of poverty study is the contribution coming from the relatively high income sectors, which are well known to demonstrate power-law scaling [37-40] of the Pareto type [41].

In summary, we have generalized the scope of our earlier work on alternative and self-consistent poverty index calculations based on mathematical models [9], now to include probabilistic measurements of the inequality index above the minimum income threshold line. The data used in our analysis were confined to years 1959-1992 but using relatively recent data from years 1993 and 2004, two not-so-close years where the fiscal dynamics are expected to be relatively independent of each other, results obtained from our model compare favorably with the representative HCI indices [11] as would be evident from the approximately parallel lines in Fig. 8. Beyond 1992, though, our model predicts a much slower precipitation of the poverty index (PI) compared to the conventional head-count index, eventually reaching an effective plateau at around year 2004-2005; we take this as a clear signature of a fast stabilizing economy (India, in this case) that seems to be justified from analysis of recent data for the year 2004-2005 [28].

This result is a major improvement over the HCI indexbased conventional studies, which fail to detect this steady state. Such a confirmation demonstrates the strength of this new model-based approach; this also emphasizes the need for such alternate poverty measures over the conventional $\mathrm{HCI} / \mathrm{PG} / \mathrm{SPG}$-based poverty evaluation. While in the absence of any dynamic models for the dominant parameters ( $V$ and $K$ ), predictions can never be "full proof," the present approach transcends all previous modeling results in the robustness of its prediction accuracy.

Given that our present model is based only on cereal consumption, it might be deemed insufficient for large-scale policy formulation as yet. The data capture from the realworld data would be hopefully much better if the model is based on a multimarket trade equilibrium using consumption expenditure data on several commodities. Related studies using kinetic models [42] as well as assuming wealth exchange as information flow in a network $[43,44]$ have recently been attempted with some success. Current works are in progress to enhance the scopes of the theoretical model even further by including nonessential foods and nonfood grain statistics in a multivariate structure $[33,34]$ to arrive at an even more robust description of consumption and income distributions. The 
future research direction here will be to integrate the present version of the consumption based description (combining all three consumption variables-basic food, nonbasic food, nonfood) to incorporate GDP time-series data, which will at least indirectly associate global trade routes, and thereby embed a global fluctuation tracking mechanism within the scope of advanced policy making. This will probabilistically foretell an endogenous policy description, ideally incorporating a social-welfare component within a capitalist architecture $[45,46]$ based on an all inclusive version of a "societydeciders model" [47], with clear indications of regions and income classes that need redistribution for inequality reduction.

Now we turn to the policy implications of our study. The literature on economics of poverty was dependent for a very long time on the preoccupation of defining who the poor were without even knowing what is poverty. This was often done based on extraneous subjective considerations as to what basket of goods constitute necessities. To add more confusion to the subjectivity, it is assumed that the so-called poverty line is objective that one can say clearly that all those whose income is just above the poverty line income are nonpoor while all those just below the poverty line income are poor. We relate our approach to the celebrated Engel's work on the economics of poverty with a hierarchy of needs and with the observations on consumption expenditure pattern of the poor and how best to improve that pattern. Once our focus is diverted to observed consumption deprivation in cereal through a distribution of consumption deprivation, and once we map such cereal deprivation distributions over time (cohort or panel data studies), we hope to identify which households are chronically deprived and perennially remain poor.

\section{ACKNOWLEDGMENTS}

A.K.C. acknowledges partial research support from Royal Society Grant No. RSO11137. Discussions with G. J. Ackland and Ewa Grela are thankfully acknowledged. T.K.K. acknowledges his debt to the coauthors of his earlier studies on Engel curve-based poverty measure, Sitaramam and Gore.
[1] E. Engel, Die Productions- und Consumtionsverhältnisse des Königreichs Sachsen, Zeitschrift des statistischen Bureaus des Königlich Sächsischen Ministerium des Inneren 8-9, 28 (1857).

[2] E. Engel, Die Lebenskosten Belgischer Arbeiter Familien friiher und jetzt, Bulletin de Institut International de Statistique 9, 1 (1895).

[3] A. Deaton, Measuring and Understanding Behavior, Welfare, and Poverty, Prize Lecture, Nobel Foundation (2015).

[4] S. Subramanian and A. Deaton, The demand for food and calories, J. Political Econ. 104, 133 (1996).

[5] K. A. Fox and T. K. Kumar, Functional economic area: Delineation and implications for economic analysis and policy, Region. Sci. Assoc. Papers 15, 57 (1965).

[6] V. Sitaramam, S. A. Paranjpe, T. K. Kumar, A. P. Gore, and J. G. Sastry, Minimum needs of poor and priorities attached to them, Econ. Pol. Weekly 31, 35/37 (1996).

[7] A. Choi and A. Moneta, Retrospective: Engel curves, J. Econ. Perspect. 24, 225 (2010).

[8] T. K. Kumar, S. K. Mallick, and J. Holla, Estimating consumption deprivation in india using survey data: A state-level rural-urban analysis before and during reform period, J. Dev. Studies 45, 441 (2009).

[9] A. K. Chattopadhyay, G. J. Ackland, and S. K. Mallick, Income and poverty in a developing economy, Europhys. Lett. 91, 58003 (2010).

[10] T. K. Kumar, A. P. Gore, and V. Sitaramam, Some conceptual and statistical issues on measurement of poverty, J. Stat. Plan. Infer. 49, 53 (1996).

[11] World Bank Poverty data, available at: http://data. worldbank.org/country/india (2016).

[12] A. K. Chattopadhyay and S. K. Mallick, Income distribution dependence of poverty measure: A theoretical analysis, Physica A 377, 241 (2007).

[13] J.-P. Bouchaud and M. Potters, in the Theory of Financial Risk and Derivative Pricing: From Statistical Physics to Risk Management (Cambridge University Press, Cambridge, 2003).
[14] I. M. Sokolov and R. Metzler, Nonuniqueness of the first passage time density of Lévy random processes, J. Phys. A 37, L609 (2004).

[15] R. Metzler, E. Barkai, and J. Klafter, Deriving fractional Fokker-Planck equations from a generalised master equation, Europhys. Lett. 46, 431 (1999).

[16] J. M. Burgers, The Non-linear Diffusion Equation: Asymptotic Solutions and Statistical Properties (Springer, Berlin, 1974).

[17] D. Colander, The systemic failure of the economics profession, Crit. Rev. 21, 249 (2009).

[18] A. K. Sen, Poverty: An ordinal approach to measurement, Econometrica 44, 219 (1976).

[19] B. S. Rowntree, Poverty: A Study in Town Life (Macmillan and Co., New York, 1901).

[20] A. Pigou and H. Dalton, The measurement of inequality of incomes, Econ. J. 30, 348 (1920).

[21] T. Goedhart et al., The poverty line: Concept and measurement, J. Human Res. 12, 503 (1977).

[22] D. Thon, On measuring poverty, Rev. Income Wealth 25, 429 (1979).

[23] N. C. Kakwani, On a class of poverty measures, Econometrica 48, 437 (1980).

[24] M. R. Carter and C. B. Barrett, The economics of poverty traps and persistent poverty: An asset based approach, J. Dev. Studies 42, 178 (2006).

[25] D. Herzer and S. Vollmer, Inequality and growth: Evidence from panel cointegration, J. Econ. Inequal. 10, 489 (2012).

[26] A. F. Shorrocks, Revisiting the sen poverty index, Econometrica 63, 1225 (1995).

[27] B. Baulch and J. Hoddinott, Economic mobility and poverty dynamics in developing countries, J. Dev. Studies 36, 1 (2000).

[28] M. H. Suryanarayana, Nutritional Norms for Poverty: Issues and Implications, available at: http://planningcommission. nic.in/reports/genrep/surya.pdf (2013).

[29] A. B. Atkinson, On the measurement of poverty, Econometrica 55, 749 (1987). 
[30] A. B. Atkinson, T. Piketty, and E. Saez, Top incomes in the long run of history, J. Econ. Literature 49, 3 (2011).

[31] X. Cirera and E. Masset, Review Article Income distribution trends and future food demand, Philos. Trans. R. Soc. London B 365, 2821 (2010).

[32] M. Ravallion, Growth, inequality and poverty: Looking beyond averages, World Dev. 29, 1803 (2001).

[33] C. Herrero, R. Martnez, and A. Villar, Multidimensional social evaluation - an application to the measurement of human development, Rev. Income Wealth 56, 483 (2010).

[34] S. Alkiere and J. Foster, Understanding and Misunderstanding of multidimensional poverty measurement, Oxford Poverty and Human Development Initiative, Working Paper 43 (2011).

[35] J. E. Foster, J. Greer, and E. Thorbecke, A class of decomposable poverty measures, Econometrica 52, 761 (1984).

[36] S. I. Denisov, W. Horsthemke, and P. Hänggi, Generalized Fokker-Planck equation: Derivation and exact solutions, Eur. Phys. J. B 68, 567 (2009).

[37] B. B. Mandelbrot, The Pareto-Levy law and the distribution of income, Int. Econom. Rev. 1, 79 (1960).

[38] E. W. Montroll and M. F. Shlesinger, Maximum entropy formalism, fractals, scaling phenomena, and 1/f noise: A tale of tails, J. Stat. Phys. 32, 209 (1983).
[39] J.-P. Bouchaud, Economics needs a scientific revolution, Nature 455, 1181 (2008).

[40] R. N. Mantegna and H. E. Stanley, Scaling behaviour in the dynamics of an economic index, Nature 376, 46 (1995).

[41] V. Pareto, La legge della domanda, Giornale degli Economisti 10, 59 (1895); English translation in Rivista di Politica Economica 87, 691 (1997).

[42] P. K. Mohanty, Generic features of the wealth distribution in ideal-gas-like markets, Phys. Rev. E 74, 011117 (2006).

[43] A. Barrat, M. Barthelemy, and A. Vespignani, in the Dynamical Processes on Complex Networks (Cambridge University Press, Cambridge, 2013).

[44] A. Carro, R. Toral, and M. S. Miguel, The noisy voter model on complex networks, Nat. Sci. Rep. 6, 24775 (2016).

[45] M. Fleurbaey and F. Maniquet, A Theory of Fairness and Social Welfare (Cambridge University Press, New York, 2011).

[46] M. L. Bertotti and G. Modanese, Microscopic models for welfare measures addressing a reduction of economic inequality, Complexity 21, 89 (2016).

[47] O. Flomenbom, The society-deciders model and fairness in nations, Biophys. Rev. Lett. 10, 157 (2015).

[48] http://www.rbi.org.in. 